\title{
Fisioterapia no tratamento da síndrome da trissomia da banda cromossômica 21 (Síndrome de Down): Revisão Sistemática
}

\author{
Physiotherapy in the treatment of chromossomal band trissomy 21 (Down Syndrome): \\ Systematic Review
}

Fisioterapia em el tratamento de la síndrome de la trisomía de la banda cromossómica 21 (Sindrome de Down): Revisión Sistemática

\begin{abstract}
Welington Jose Gomes Pereira ${ }^{1 *}$, Cristiane Gonçalves Ribas ${ }^{2}$, Edson Cit Junior ${ }^{3}$, Simone Cristina Pires Domingos ${ }^{3}$, Taina Gomes Valerio ${ }^{3}$, Thayna Aquino Gonçalves ${ }^{3}$.
\end{abstract}

\section{RESUMO}

Objetivo: Neste artigo cientifico apresenta-se um estudo do tipo revisão de literatura que teve como objetivo principal compreender quais as principais técnicas fisioterapêuticas utilizadas para o tratamento da Síndrome de Down (SD) e os principais resultados obtidos nestas pesquisas. Métodos: Estudo exploratório do tipo revisão de literatura, sendo aplicados os descritores "Síndrome de Down" e "Fisioterapia" em português e inglês nas seguintes bases de dados: CAPES, Scielo, Medline e LILACS, na linha de tempo dos últimos 10 anos. Resultados: Nas respectivas bases de dados foi computado 70 pesquisas com estes descritores nos últimos 10 anos, porém, após os critérios de inclusão e exclusão foram constatados que apenas 10 artigos apresentaram dados qualitativos ou quantitativos sobre a prática da fisioterapia nos tratamentos das disfunções musculares ou motoras em portadores de (SD). Os autores pesquisados demonstraram uma tendência de pesquisa voltada para lactentes, sendo que dos 10 artigos 9 eram voltadas para as crianças e apenas 1 pesquisa para adolescentes e adultos, demonstrando uma carência para o segmento da terceira idade. Considerações Finais: Os resultados das pesquisas destes 10 artigos apontam que tratamentos fisioterapêuticos que aplicam protocolos multissensoriais demonstram bons resultados na reabilitação motora, entretanto, os fisioterapeutas ainda utilizam técnicas consideradas mais tradicionais como Bobath e Kabat.

Palavras-chave: Síndrome de Down, Fisioterapia, Trissomia do Cromossomo 21.

\begin{abstract}
Objective: This scientific article presents a literature review study that aimed to understand the main physiotherapeutic techniques used for the treatment of Down Syndrome (DS) and the main results obtained in these researches. Methods: An exploratory study of the literature review type, applying the descriptors "Down Syndrome" and "Physiotherapy" in Portuguese and English in the following databases: CAPES, Scielo, Medline and LILACS, in the timeline of the last 10 years. Results: In the respective databases, 70 researches with these descriptors were computed in the last 10 years, but after the inclusion and exclusion criteria it was found that only 10 articles presented qualitative or quantitative data on the practice of physiotherapy in the treatment of muscle dysfunction or motor carriers (SD). The authors surveyed demonstrated a research trend towards infants, and of the 10 articles 9 were aimed at children and only 1 research for adolescents and adults, showing a lack for the elderly segment. Final Considerations: The research results of these 10 articles indicate that physiotherapeutic treatments that apply multisensory protocols show good results in motor rehabilitation, however, physiotherapists still use techniques considered more traditional as Bobath and Kabat.
\end{abstract}

Key words: Down's syndrome, Physiotherapy, Chromosome Trisomy 21.

\footnotetext{
${ }^{1}$ PPGEB - Programa de Pós-Graduação em Engenharia Biomédica, Universidade Tecnológica Federal do Paraná (UTFPR), Curitiba/PR.

2 Pontifícia Universidade Católica, Curitiba/PR.

3 Centro Universitário Dom Bosco (UniDBSCO), Curitiba/PR. *E-mail: welington.pereira@compagas.com.br
} 


\section{RESUMEN}

Objetivo: este artículo científico presenta un estudio de revisión de la literatura que tuvo como objetivo comprender las principales técnicas fisioterapéuticas utilizadas para el tratamiento del síndrome de Down (DS) y los principales resultados obtenidos en estas investigaciones. Métodos: estudio exploratorio del tipo de revisión de la literatura, que aplica los descriptores "Síndrome de Down" y "Fisioterapia" en portugués e inglés en las siguientes bases de datos: CAPES, Scielo, Medline y LILACS, en la línea de tiempo de los últimos 10 años. Resultados: En las bases de datos respectivas, se calcularon 70 investigaciones con estos descriptores en los últimos 10 años, pero después de los criterios de inclusión y exclusión se encontró que solo 10 artículos presentaron datos cualitativos o cuantitativos sobre la práctica de la fisioterapia en el tratamiento de la disfunción muscular o autotransportes (SD). Los autores encuestados demostraron una tendencia de investigación hacia los bebés, y de los 10 artículos, 9 estaban dirigidos a niños y solo 1 investigación para adolescentes y adultos, lo que muestra una falta para el segmento de personas mayores. Consideraciones finales: Los resultados de la investigación de estos 10 artículos indican que los tratamientos fisioterapéuticos que aplican protocolos multisensoriales muestran buenos resultados en la rehabilitación motora, sin embargo, los fisioterapeutas siguen utilizando técnicas consideradas más tradicionales como Bobath y Kabat.

Palabras clave: Sindrome de Down, Fisioterapia, Trisomía del Cromosoma 21.

\section{INTRODUÇÃO}

Segundo Moreira L et al. (2000), a trissomia da banda cromossômica 21q22 é um dos fatores responsáveis pela condição genética da Síndrome de Down (SD), síndrome esta que pode ocasionar uma série de transtornos físicos e mentais aos seus portadores, dentre elas a deficiência mental. Para Shepherd R (2002), a SD é considerada uma das causas mais frequentes da incapacidade mental nos seres humanos, comprometendo a capacidade funcional e intelectual destas pessoas.

De acordo com Santos JA et al. (2006), a SD é uma condição genética que assola a espécie humana desde os seus primórdios, porém, somente em meados do século XIX que a humanidade começou a compreender melhor sobre esta síndrome, graças devido as pesquisas efetuadas pelo cientista John Langdon Down, que elaborou uma série de estudos clínicos relatando diversos achados científicos, mesmo durante um período histórico no qual a ciência iniciava os seus estudos sobre campo da genética.

Entretanto, Bissoto ML (2005) relata que devido à falta de conhecimento de genética por parte de Langdon, este pesquisador efetuou uma associação errônea da SD com as características étnicas das pessoas, relatando que a SD estava sujeita a grupos de indivíduos considerados de raças inferiores, algo comum e tendência da época. Para Santos JA et al. (2006), foi durante este período histórico que diversos autores utilizaram o termo mongoloide aos portadores da SD, definição esta que foi aplicada até o final da década de 1980 , quando a classe cientifica aboliu este termo e passou a utilizar o conceito da trissomia do cromossomo 21 e a terminologia da Síndrome de Down.

Para Silva NLP e Dessen MA (2002), devido a SD possuir características físicas e mentais em graus variados em seus portadores, inicialmente rotulavam estes indivíduos como incapazes intelectualmente, somente após o advento das pesquisas genéticas foi possível compreender mais sobre esta síndrome e principalmente os fatores associados que a causavam.

Ainda segundo Silva NLP e Dessen MA (2002), desde o relato cientifico efetuado por Langdon em meados do século XIX, somente em 1932 com a pesquisa elaborada pelo cientista holandês Waardenburg que foi relatado a hipótese da SD estar relacionada com alguma alteração cromossômica dentre elas a trissomia, fato este comprovado apenas em 1959 nas pesquisas efetuadas por Jerone Lejeune e Patricia A. Jacobs, sendo esta data um marco histórico para as pesquisas sobre SD.

De acordo com Bissoto ML (2005), após a comprovação cientifica que a SD estava relacionada diretamente com a trissomia do cromossomo 21, diversas pesquisas cientificas foram efetuadas a fim de compreender mais sobre esta síndrome como também propor possíveis tratamentos clínicos aos seus portadores. 
Segundo Schwartzman JS (1999), pouco se conhece sobre os fatores que ocasionam a SD podendo ser fatores endógenos (mais frequentes associados a idade da mãe, pois os envelhecimentos dos óvulos podem aumentar o risco da SD em nascidos), e fatores exógenos (associados ao uso de substancias tóxicas ou contaminantes).

Para Silva NLP e Dessen MA (2002), estas alterações endógenas ou exógenas ocasionam um desiquilíbrio na constituição cromossômica, neste caso em específico, a presença de um cromossomo 21 extra sendo caracterizada uma trissomia simples.

Porém esta alteração no cromossomo podem ser caracterizadas por uma translocação ou um mosaico, pois de acordo com Schwartzman JS (1999), na translocação do cromossomo 21 adicionais está fundido outro autossomo sendo estas alterações comuns ocorrerem nos cromossomos 14 e 21.

Ainda segundo Silva NLP e Dessen MA (2002), a resultante destas alterações é uma braquicefalia nos portadores de SD descrita por um diâmetro fronto-occipital pequeno e fissuras palpebrais com inclinação superior, pregas epicânticas, base nasal achatada e hipoplasia, características físicas predominantes nos portadores de SD.

Para Moreira L et al. (2000), além dos aspectos cognitivos, intelectuais e motores, a SD pode ocasionar cardiopatias congênitas, hipotonia, problemas de audição e visão, como também alterações na coluna cervical e distúrbios da tireoide.

Estas patologias relatadas por Moreira $L$ et al. (2000), reduzem a expectativa de vida em seus portadores, sendo que no início do século XX a expectativa de vida dos portadores de SD eram abaixo dos 40 anos, entretanto, de acordo com os estudos efetuados por Silva NLP e Dessen MA (2002) e Bissoto ML (2005) com advento de novas tecnologias e tratamentos de saúde entre elas a fisioterapia a expectativa e qualidade de vida destes indivíduos aumentaram consideravelmente, sendo comum portadores de SD alcançarem idades superiores aos 60 anos,

Porém, a incidência de novos casos de SD veem aumentando consideravelmente nas últimas décadas, pois de acordo com a National Down Syndrome Society (NDSS), nos Estados Unidos 1 para cada 691 nascidos possuem esta síndrome o que equivale uma população de cerca de 400 mil pessoas nos EUA, já no Brasil, o censo efetuado pelo Instituto Brasileiro de Geografia e Estatística (IBGE) em 2010 estimou uma população em cerca de $23,9 \%$ de indivíduos com alguma deficiência intelectual, entretanto, não existe uma estatística especifica para apontar quantos destes indivíduos possuem a SD, entretanto, segundo a Federação Brasileira das Associações de Síndrome de Down (FBASD) estima-se que existam uma relação base de 1 para cada 700 nascidos no Brasil, o que representam uma população entorno de 270 mil pessoas com SD.

Para Alberto PCD (2014), devido a este aumento da incidência de novos casos de SD é fundamental políticas públicas de saúde voltadas para estes indivíduos, buscando melhorar a qualidade e longevidade de vida, sendo recomendado tratamentos de fisioterapia.

Coppede AC et al. (2012) complementa as considerações efetuadas por Alberto PCD (2014), relatando que a fisioterapia proporciona aos portadores da SD tratamentos paras as condições musculares, respiratórias e funcionais, tratamentos estes que podem melhorar significativamente as funcionalidades motoras nestes indivíduos.

Por estes motivos relatados anteriormente, esta pesquisa cientifica justifica-se pela necessidade da compreensão das principais técnicas de tratamentos fisioterapêuticos aplicados aos portadores desta síndrome.

O objetivo geral deste artigo é pesquisar quais foram os principais achados científicos nos últimos 10 anos referente aos tratamentos fisioterapêuticos, afim de verificar quais as melhores técnicas e os seus respectivos resultados, deste modo, o resultado final desta pesquisa será a elaboração de um panorama cientifico que servirá de base para pesquisas futuras sobre este tema. 


\section{MÉTODOS}

Esta pesquisa científica trata-se de um estudo descritivo de análise documental com uma abordagem qualitativa do tipo revisão bibliográfica, sendo adaptado o método científico proposto por Pereira WJG et al (2018) que estrutura e organiza uma revisão de literatura em 4 etapas de filtros.

As premissas do método cientifico foram as seguintes: Efetuar uma pesquisa sistemática de literatura utilizando os descritores: "Síndrome de Down" AND "Fisioterapia" em português e "Down's Syndrome" AND "Physiotherapy" em inglês, a escolha dos descritores seguiram as especificações segundo as normas de descritores em ciências da saúde (DesCS).

A pesquisa sistemática ocorreu nas seguintes bases de dados: CAPES, SciELO, LILACS e Medline. Os critérios de inclusão adotados foram: Artigos científicos completos com publicação entre janeiro de 2008 a dezembro de 2018 nas bases de dados citadas. Já os critérios de exclusão foram: Artigos em idiomas diferentes de português e inglês, resumos, revisões de literatura, cartas aos editores, e artigos não abertos para o público.

\section{RESULTADOS}

Após as 4 etapas de filtragem da pesquisa sistemática foram selecionados inicialmente 70 publicações, porém, após os critérios de exclusão apenas 10 pesquisas foram selecionadas por terem dados qualitativos ou quantitativos sobre aplicação da fisioterapia nos tratamentos em portadores de SD.

Um resultado preliminar interessante é a concentração destas pesquisas em um mesmo periódico, sendo constatado 6 publicações das 10 selecionadas periódico Revista Fisioterapia em Movimento, demonstrando que esta revista possui uma temática forte sobre este tema atraindo a maioria dos autores.

Os resultados obtidos em cada etapa do processo metodológico foram resumidos e diagramados na Figura 1 para melhor visualização, como também a tabela resumida com os principais achados clínicos destes artigos na Tabela 1. 


\section{Revista Eletrônica Acervo Saúde / Electronic Journal Collection Health ISSN 2178-2091}

Figura 1 - Fluxograma dos resultados da pesquisa sistemática. FISIOTERAPIA NO TRATAMENTO DA SINDROME DA TRISSOMIA DA BANDA CROMOSSÔMICA 21 (SINDROME DE DOWN): REVISÃO SISTEMÁTICA

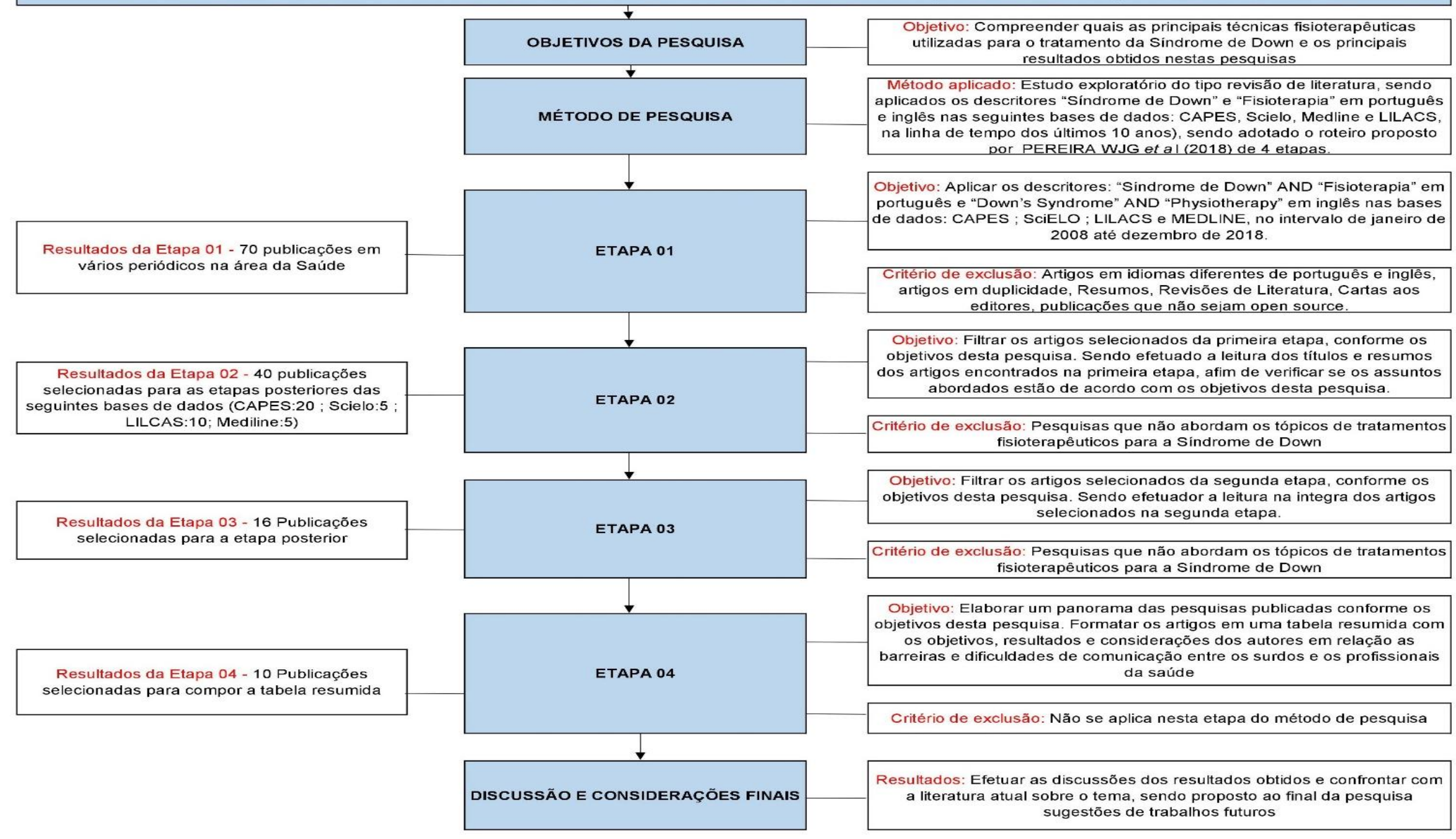

Fonte: Os autores (2019). 
Tabela 1- Resumo dos 10 artigos publicados entre janeiro de 2008 e dezembro de 2018.

\section{Título}

\begin{tabular}{|c|c|c|c|}
\hline Titulo & Autores & Periodico & Objetivos da pesquisa \\
\hline $\begin{array}{l}\text { Aquisição do sentar } \\
\text { independente na Síndrome de } \\
\text { Down } \\
\text { utilizando o balanço }\end{array}$ & $\begin{array}{l}\text { GODZICKI, B et } \\
\text { al. }\end{array}$ & $\begin{array}{l}\text { Revista Fisioterapia } \\
\text { Movimento, ano } \\
\text { publicação: } 2010\end{array}$ & $\begin{array}{l}\text { Avaliar a eficácia do tratamento } \\
\text { por meio do balanço para a } \\
\text { aquisição do sentar } \\
\text { independente em crianças com } \\
\text { Síndrome de Down. }\end{array}$ \\
\hline $\begin{array}{l}\text { Força de preensão e destreza } \\
\text { manual na criança com } \\
\text { Síndrome de Down }\end{array}$ & PRIOSTI, PA et al. & $\begin{array}{l}\text { Revista Fisioterapia e } \\
\text { Pesquisa, ano de } \\
\text { publicação: } 2013\end{array}$ & $\begin{array}{l}\text { Oreste estudo foi analisar a } \\
\text { destivo } \\
\text { correlação entre força de } \\
\text { preensão e destreza manual em } \\
\text { crianças com SD e crianças } \\
\text { saudáveis com idade entre } 7 \text { e } 9 \\
\text { anos. }\end{array}$ \\
\hline $\begin{array}{l}\text { A existência de alterações } \\
\text { neurofisiológicas pode auxiliar } \\
\text { na compreensão } \\
\text { do papel da hipotonia no } \\
\text { desenvolvimento motor dos } \\
\text { indivíduos } \\
\text { com síndrome de Down? } \\
\end{array}$ & $\begin{array}{l}\text { CORREAA, JCF et } \\
\text { al. }\end{array}$ & \begin{tabular}{ll} 
Revista & \multicolumn{2}{c}{ Fisioterapia } \\
pesquisa, ano de & ano de \\
publicação: 2011 &
\end{tabular} & $\begin{array}{l}\text { Analisar e verificar a existência de } \\
\text { alterações neurofisiológicas nos } \\
\text { indivíduos com SD, como a } \\
\text { hiporreflexia, estática e dinâmica. }\end{array}$ \\
\hline $\begin{array}{ll}\text { Hidrocinesioterapia } & \text { no } \\
\text { tratamento fisioterapêutico } & \text { de } \\
\text { um lactente com Síndrome de } \\
\text { Down: estudo de caso }\end{array}$ & TOBLE, AM et al. & $\begin{array}{l}\text { Revista Fisioterapia em } \\
\text { Movimento, ano de } \\
\text { publicação: } 2013\end{array}$ & $\begin{array}{l}\text { Investigar a eficácia da hidro } \\
\text { cinesioterapia } \\
\text { como método complementar de } \\
\text { tratamento fisioterapêutico na } \\
\text { aquisição de habilidades motoras } \\
\text { grossas. }\end{array}$ \\
\hline $\begin{array}{l}\text { Profile of physiotherapy } \\
\text { intervention for Down syndrome } \\
\text { children }\end{array}$ & $\begin{array}{l}\text { MORAIS, KDW et } \\
\text { al. }\end{array}$ & $\begin{array}{l}\text { Revista Fisioterapia em } \\
\text { Movimento, ano de } \\
\text { publicação: } 2016\end{array}$ & $\begin{array}{l}\text { Estudo teve como objetivo } \\
\text { investigar o perfil do atendimento } \\
\text { fisioterapêutico às crianças com } \\
\text { SD, nos primeiros três anos de } \\
\text { idade. }\end{array}$ \\
\hline $\begin{array}{l}\text { Effect of hippotherapy in the } \\
\text { global motor coordination } \\
\text { in individuals with Down } \\
\text { Syndrome }\end{array}$ & COSTA, VSF et al & $\begin{array}{l}\text { Revista Fisioterapia em } \\
\text { Movimento, ano de } \\
\text { publicação: } 2017\end{array}$ & $\begin{array}{l}\text { Analisar os efeitos de um } \\
\text { programa } \\
\text { de Equoterapia sobre as variáveis } \\
\text { de coordenação motora global em } \\
\text { indivíduos com SD de ambos os } \\
\text { gêneros e comparar indivíduos } \\
\text { com a mesma síndrome que não } \\
\text { praticam Equoterapia. }\end{array}$ \\
\hline
\end{tabular}

\section{Método de Pesquisa}

Aplicado um tratamento em crianças entre 6 e 7 meses de vida com duração de 30 minutos em cada sessão, em um balanço composto por plataforma revestida com Etil vini acetato, realizando-se deslocamentos ineares no sentido ântero-posterior.

Participaram 26 crianças com SD, de ambos os sexos. A avaliação da força de preensão foi realizada com dinamômetro Jamar, e a da destreza manual pelo Teste Caixa e Blocos.

Estudo de caráter transversa controlado, foi aplicado em 24 voluntários com diagnóstico de SD 25 voluntários sem SD como grupo controle.

\section{Pesquisa exploratória, tendo como} participante 1 lactente com SD sendo submetido a um protocolo de desenvolvimento motor e avaliado por meio da Alberta Infant Motor Scale (AIMS) durante um período de 24 sessões com duração de 1 hora cada. Estudo qualitativo por meio de
coletadas de dados realizadas em entrevistas com fisioterapeutas.

Estudo exploratório, contendo 4 participantes sendo 20 que praticavam Equoterapia (GE) e 21 que não praticavam Equoterapia (GC). Utilizou-se

teste körperkoordinations test für Kinder (KTK)

\section{Principais Resultados}

Observou-se que, quando estimuladas precocemente por meio do balanço, essas crianças

adquiriram o sentar antes do tempo descrito pela literatura.

O Grupo SD apresentou desempenho inferior tanto na força de preensão quanto na destrezar manual, quando comparado ao GC; não houve correlação significativa entre a força de preensão e a destreza manual no Grupo SD.

Neste estudo foi verificado a existência de diminuição da velocidade de condução do potencial de ação, de forma crônica, podem ocasionar prejuízo no controle sensório-moto dos músculos, e consequentemente, hipotonia desses indivíduos.

A hidro terapêutica propiciou a estimulação sensorial e o aprimoramento do controle e do fortalecimento

dos músculos do tronco do lactente com SD refletindo melhor desempenho motor nas posturas antigravitacionais, prona e sentada. Os $\begin{array}{ccc}\text { resultados } & \text { indicam }\end{array}$ tratamentos em crianças com SD é o método Bobath, com protocolo de 2 vezes por semana com duração em média de 30 minutos.

Comparando os grupos observou-se diferença significativa $(p<0,01)$ para 0 Quociente Motor da Tarefa de Salto lateral, o GE apresentando melhor escore $(114,10)$ em relação ao GC $(88,47)$, melhores resultados na coordenação motora global. 


\begin{tabular}{|c|c|c|c|c|c|}
\hline 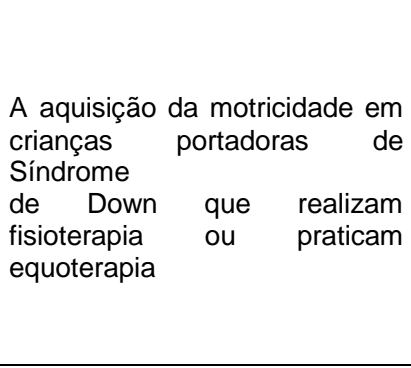 & $\begin{array}{l}\text { TORQUATO, JA } \\
\text { et al. }\end{array}$ & $\begin{array}{l}\text { Revista Fisioterapia em } \\
\text { Movimento, ano de } \\
\text { publicação: } 2017\end{array}$ & $\begin{array}{l}\text { Verificar a aquisição de marcos } \\
\text { motores em crianças portadoras } \\
\text { de SD } \\
\text { que realizam a equoterapia ou } \\
\text { fisioterapia convencional. }\end{array}$ & \begin{tabular}{|l|} 
Estudo transversal que aplicado em \\
33 indivíduos portadores de SD com \\
idade entre 4 e 13 anos, de ambos os \\
sexos, divididos \\
em 2 grupos: Grupo 1 - equoterapia; \\
Grupo 2 - fisioterapia em solo. A \\
motricidade global, o equilíbrio \\
estático ero \\
o dinâmico foram avaliados com uso \\
da Escala de Desenvolvimento Motor \\
(EDM).
\end{tabular} & $\begin{array}{l}\text { As crianças } \\
\text { que realizam fisioterapia apresentam melhor } \\
\text { equilíbrio estático e dinâmico do que } \\
\text { indivíduos que realizam equoterapia, A } \\
\text { fisioterapia convencional teve influência } \\
\text { positiva na obtenção das aquisições } \\
\text { motoras e do equilíbrio estático e dinâmico em } \\
\text { portadores de SD. }\end{array}$ \\
\hline $\begin{array}{l}\text { Desempenho motor fino e } \\
\text { funcionalidade } \\
\text { crianças com síndrome de } \\
\text { Down }\end{array}$ & $\begin{array}{l}\text { COPPEDE, AC et } \\
\text { al. }\end{array}$ & $\begin{array}{l}\text { Revista Fisioterapia } \text { e } \\
\text { Pesquisa, ano de } \\
\text { publicação: } 2012\end{array}$ & $\begin{array}{l}\text { O objetivo do estudo foi comparar } \\
\text { crianças } \\
\text { com SD e crianças típicas quanto } \\
\text { ao } \\
\text { desempenho motor fino, avaliado } \\
\text { pela Bayley Scales of Infant and } \\
\text { Toddler Development - Third } \\
\text { Edition (BSITD-III). }\end{array}$ & $\begin{array}{l}\text { Estudo transversal do tipo caso- } \\
\text { controle. Participaram } 24 \text { crianças de } \\
\text { ambos os gêneros, nascidas a termo, } \\
\text { e com peso adequado para idade } \\
\text { gestacional, sendo } 12 \text { crianças } \\
\text { pertencentes ao grupo típico (T). }\end{array}$ & $\begin{array}{l}\text { As crianças com SD apresentaram perfil } \\
\text { motor fino e funcional inferiores às crianças } \\
\text { com desenvolvimento } \\
\text { típico; porém, o desempenho funcional do } \\
\text { grupo SD esteve adequado segundo o } \\
\text { esperado para a faixa etária. }\end{array}$ \\
\hline $\begin{array}{l}\text { Self-controlled feedback } \\
\text { enhances learning in } \\
\text { adults with Down syndrome }\end{array}$ & $\begin{array}{l}\text { CHIVIACOWSKY, } \\
\text { S et al. }\end{array}$ & $\begin{array}{l}\text { Brazilian Journal of } \\
\text { Physical Therapy: } 2012\end{array}$ & $\begin{array}{l}\text { Examinar se os benefícios do } \\
\text { feedback autocontrolado para a } \\
\text { aprendizagem em adultos que } \\
\text { apresentam a SD. }\end{array}$ & 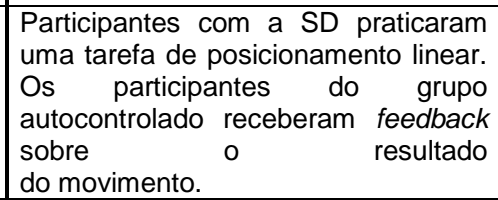 & $\begin{array}{l}\text { Feedback autocontrolado melhora a } \\
\text { aprendizagem motora em sujeitos com a SD. }\end{array}$ \\
\hline $\begin{array}{l}\text { Avaliação do equilíbrio estático } \\
\text { de } \quad \text { crianças } \\
\text { e adolescentes com síndrome } \\
\text { de Down }\end{array}$ & $\begin{array}{l}\text { MENEGHETTI, } \\
\mathrm{CHZ} \text { et al. }\end{array}$ & $\begin{array}{l}\text { Brazilian Journal of } \\
\text { Physical Therapy: } 2009\end{array}$ & $\begin{array}{l}\text { Avaliar o equilíbrio estático de } \\
\text { crianças e adolescentes com SD } \\
\text { pelariofotogrametria } \\
\text { computadorizada }\end{array}$ & $\begin{array}{|lrr|}\text { Participaram } 11 \text { crianças } & e \\
\text { adolescentes com SD e } 14 \text { crianças e } & \\
\text { adolescentes de ambos os gêneros, } \\
\text { neurologicamente normais que } \\
\text { compuseram o grupo controle, } & \text { O } \\
\text { instrumento utilizado foi } & \text { a } \\
\text { Biofotogrametria Computadorizada. }\end{array}$ & $\begin{array}{l}\text { As crianças e adolescentes com SD oscilaram } \\
\text { mais que as do grupo controle e, quaando a } \\
\text { informação visual foi manipulada, as as } \\
\text { oscilações ântero-posterior e latero-lateral } \\
\text { mostraram a existência de diferenças. }\end{array}$ \\
\hline
\end{tabular}

Fonte: Os Autores (2019). 


\section{DISCUSSÃO}

Nesta revisão sistemática foi possível observar que as principais pesquisas publicadas e armazenadas nas bases de dados (CAPES, Scielo, Medline e LILACS), concentraram-se no estudo do atraso motor em portadores de SD, em especifico crianças em fase lactentes e na primeira infância, sendo constatado poucos estudos para portadores de SD na fase adulta.

Outro aspecto importante, é a concentração das pesquisas no campo de estudo da fisioterapia motora, sendo poucos estudos abordando aspectos em outros segmentos da fisioterapia, como por exemplo a fisioterapia respiratória, cardiovascular e cognitiva, seguindo um sentido contrário do que descrito por Moreira $\mathrm{L}$ et al. (2000), que relatam que os efeitos da SD podem causar distúrbios na respiração, sistema circulatório, audição e visão.

Entretanto, segundo Morais KDW et al. (2010), as intervenções fisioterapêuticas oferecidas aos portadores de SD vão além da área médica e de saúde, pois as necessidades destes indivíduos possuem um caráter interdisciplinar que envolvem diversas áreas do conhecimento, entre elas: Medicina, psicologia, fonoaudiologia, fisioterapia, terapia ocupacional e educadores em geral. Ainda segundo Morais KDW et al. (2010), atuação destes profissionais em conjunto tem como objetivo principal melhorar a capacidade física e psicológica dos portadores de SD, a fim de proporcionar uma qualidade de vida a estes indivíduos.

No que tange a fisioterapia, no estudo efetuado por Coppede AC et al. (2012), que avaliou o desenvolvimento motor de um grupo de crianças com SD em comparação com outro grupo sem a síndrome foi constatado estatisticamente que crianças com SD apresentam um desempenho motor fino inferior as crianças sem a síndrome.

Ainda segundo Coppede AC et al. (2012), os portadores de SD possuem dificuldades nas habilidades que envolvam o controle e planejamento motor, percepção visual e integração visomotora, o que podem afetar diretamente nas habilidades funcionais de autocuidado na fase adulta.

Estes dados apontados por Coppede AC et al. (2012), estão alinhados com os resultados obtidos nos estudos efetuados por Corrêa JCF et al. (2011) e Meneghetti CHZ et. al (2009), que relatam que a resposta reflexa dos pacientes com SD é menor do que em comparação aos indivíduos sem a síndrome.

Ainda segundo o estudo efetuado por Corrêa JCF et al. (2011), este atraso do reflexo motor ocorre devido a ação do estimulo acontecer em nível medular não tendo a influência do córtex cerebral que efetua o controle e ajustes dos movimentos finos, que em associação ao processo de hipotonia que a SD ocasiona em seus portadores fazem com que os músculos realizem uma contração mais lenta ou ineficaz prejudicando consequentemente os movimentos finos.

Este comprometimento no processo de estimulo e controle motor nos portadores da SD acarreta em um comprometimento na força muscular, pois segundo o estudo efetuado por Priosti PA et al. (2009), que avaliou a destreza manual de crianças com SD e sem a síndrome foi constatado que os portadores de SD possuem um desempenho inferior no que tange a força de pressão em comparação com o outro grupo, a falta desta força muscular tendem a afetar o equilíbrio postural estático ou dinâmico.

Coppede AC et al. (2012), complementa as considerações efetuadas por Priosti PA et al. (2009), relatando que os efeitos motores ocasionados pela SD ocorrem em maior ênfase nos primeiros meses de vida, comprometendo o desenvolvimento motor nas crianças.

Para o tratamento destas disfunções motoras, principalmente aquelas relacionadas com a capacidade de equilíbrio de tronco e postural, Godzicki B et al. (2010), relatam em seu estudo que uma estratégia para efetuar estímulos neuromusculares é a utilização de protocolos de exercícios multissensoriais que envolvam a percepção visual e motora, tendo como foco melhorar a capacidade postural.

Neste estudo efetuado por Godzicki B et al. (2010), foi aplicado um protocolo de exercícios de estímulos que adotaram a utilização de uma plataforma em balanço e um tapete texturizado em vinil tendo como objetivo melhorar a capacidade de controle do tronco e auxiliar as crianças no processo de sentar independente, com 
base neste protocolo, estes autores obtiveram resultados satisfatórios após o período de intervenção nas crianças pesquisadas, pois elas melhoraram significativamente os reflexos primitivos de proteção, de controle postural e cognitivos, demonstrando que exercícios multissensoriais são benéficos no processo de reabilitação.

Estes resultados apontados por Godzicki B et al. (2010), vão de encontro com os resultados obtidos na pesquisa efetuada por Torquato JA et al (2013), que aplicaram um protocolo de exercícios multissensoriais para aquisição de motricidade em crianças com SD por meio da equoterapia em comparação com crianças que efetuaram a fisioterapia convencional como meio de tratamento.

Neste estudo, os resultados apontaram que a equoterapia proporcionou uma melhora nos marcos motores de equilíbrio estático e dinâmico, porém, em comparação com a fisioterapia convencional a equoterapia alcançou resultados inferiores no que tange o desenvolvimento motor, entretanto, não ficou evidenciado neste estudo outros aspectos importantes nos tratamentos que são a satisfação e aceitação por parte dos pacientes como também a questão da melhora cognitiva (TORQUATO JA et al.,2013).

Por este motivo, o uso da fisioterapia convencional por parte dos fisioterapeutas nos tratamentos da SD é mais comum nestes casos, pois segundo o estudo efetuado por Morais KDW et al. (2010), que efetuou uma pesquisa qualitativa com diversos fisioterapeutas perguntando qual o melhor método aplicado para 0 tratamento das disfunções motoras ocasionadas pela SD, obteve-se como resultado da pesquisa que 0 método mais aplicado pelos entrevistados é o Bobath por ser um tratamento neuroevolutivo que tem como objetivo central a inibição dos padrões das atividades reflexas anormais que em associação com técnicas de estimulação tátil proprioceptivas que promovem um ajustamento dos movimentos intrínsecos e automáticos.

Ainda segundo Morais KDW et al. (2010), outra técnica fisioterapêutica convencional aplicada é o método Kabat que é uma técnica de Facilitação Neuromuscular Proprioceptiva (FNP) e tem como finalidade melhorar a coordenação motora por meio da reeducação seletiva de cada elemento motor, possuindo como objetivo específico em aumentar ou recuperar a força muscular dos membros como também desenvolver a coordenação motora nos pacientes.

Para Torquato et al. (2010), no processo de desenvolvimento motor em crianças com SD o mais importante não é propriamente a técnica multissensorial a ser aplicada, e sim quando ser utilizada e a duração dos tratamentos, pois segundo estes autores o desenvolvimento motor nos portadores de SD é mais demorado em comparação aos indivíduos sem a síndrome, sendo neste caso, fundamental o início do tratamento nos primeiros dias de vida e seguir com este tratamento por um longo período até alcançarem os objetivos de tratamento.

Ainda segundo Torquato JA et al (2013), os fisioterapeutas utilizam outras técnicas de tratamentos além da fisioterapia convencional, entre elas a hidrocinesioterapia, pois esta técnica consiste em uma terapia aquática que utiliza as propriedades físicas da água e sua capacidade térmica para promover o bem estar físico, como também no processo de reabilitação cardiovascular, relaxamento e diminuição do tônus muscular, criando assim um ambiente estimulante para o paciente.

Na pesquisa efetuada por Toble AM et al (2013), que efetuou um estudo com um grupo de lactentes com SD no qual foram submetidos a um protocolo de intervenção utilizando a técnica de hidroterapia, obteve-se uma melhora significativa no desenvolvimento das habilidades motoras grossas, como também melhora na capacidade auditiva.

Ainda segundo Toble AM et al (2013), um dos fatores destes resultados obtidos pode ser explicado pelos princípios físicos da água, que criam um ambiente propicio para estimulação sensorial e aprimoramento no controle e do fortalecimento dos músculos do tronco, o que melhoraram nos indicadores das posturas antigravitacionais de prona e sentada nos pacientes.

Entretanto, os tratamentos fisioterapêuticos não concentram-se apenas em protocolos de intervenção motora sensorial, existem outros aspectos fundamentais que podem influenciar no processo de reabilitação entre eles a educação e orientação aos portadores de SD, neste caso, o estudo efetuado por Chiviacowsky 
$S$ et al. (2012) que aplicaram a técnica de feedback auto controlado em um grupo de jovens e adultos com SD e sem síndrome obtiveram como resultado a melhora dos movimentos em exercícios após terem as orientações corretas para a execução, demonstrando que a orientação verbal podem influenciar diretamente na melhora motora dos pacientes.

Por fim, nesta pesquisa sistemática ficou evidenciado que existe uma gama de tratamentos fisioterapêuticos direcionados para os portadores de SD, entretanto, cada modalidade e técnica a ser aplicada tendem a variar conforme o grau de comprometimento dos indivíduos sendo recomendada a escolha dos protocolos de atendimento conforme as necessidades especificam dos pacientes. Porém, outro aspecto apontado neste estudo é o fato da classe cientifica e acadêmica focar os seus esforços e pesquisas no campo da reabilitação motora, deixando um pouco de lado outras áreas da fisioterapia no tratamento da SD.

\section{CONCLUSÃO}

Os tratamentos fisioterapêuticos voltados aos portadores da SD podem melhorar a qualidade e expectativa de vida nestes indivíduos, entretanto, as necessidades dos portadores desta síndrome envolvem diversos aspectos físicos, fisiológicos e psicológicos o que demandam atenção de uma equipe multidisciplinar. Neste estudo, ficou evidenciado uma tendência de pesquisa pela classe acadêmica sobre este tema, em principal, as questões que envolvam o processo de reabilitação motora, sendo poucos estudos que abordam outras características, entre elas as questões respiratórias, cardiovascular e cognitiva. A falta de pesquisas sobre estes temas relatados anteriormente demonstra uma carência e falta de interesse dos pesquisadores em buscarem novas técnicas e meios de tratamentos destas disfunções. Outro aspecto evidenciado nesta pesquisa é o foco dos estudos serem na fase lactente ou infantil em portadores de SD, sendo que a grande maioria dos estudos são voltados para estes grupos, não sendo constatado nenhum estudo direcionado para a terceira idade, grupo este de indivíduos que podem apresentar necessidades especifica diferentes das outras fases da vida. Entretanto, no que tange os resultados relacionados ao processo de reabilitação motora ficou evidenciado que exercícios multissensoriais melhoram significativamente o processo de reabilitação, independentemente das técnicas abordadas (Hidrocinésioterapia, Equoterapia, fisioterapia convencional), porem, devido aos poucos estudos publicados relacionados a novas técnicas de tratamento a classe fisioterapêutica ainda utilizam técnicas consideradas mais conservadoras entre elas os métodos Bobath e Kabat.

\section{REFERÊNCIAS}

1. ALBERTO PCD. Sindrome de down. Revista de Actualización Clínica, v. 46, n. 2304-3768, 2014.

2. BISSOTO ML. Desenolvimento cognitivo e o processo de aprendizagem do portador de síndrome de Down: revendo concepções e perspectivas educacionais. Ciências \& Cognição, v. 4, 2005.

3. CORRÊA JCF et al. A existência de alterações neurofisiológicas pode auxiliar na compreensão do papel da hipotonia no desenvolvimento motor dos indivíduos com síndrome de Down?. Fisioterapia e Pesquisa, v. 18, n. 4, p. 377-381, 2011.

4. CHIVIACOWSKY S et al. Self-controlled feedback enhances learning in adults with Down syndrome. Brazilian Journal of Physical Therapy, v. 16, n. 3, p. 191-196, 2012.

5. COPPEDE AC et al. Desempenho motor fino e funcionalidade em crianças com síndrome de Down. Fisioterapia e Pesquisa, v. 19, n. 4, p. 363-368, 2012.

6. COSTA VS et al. Effect of hippotherapy in the global motor coordination in individuals with Down Syndrome. Fisioterapia em Movimento, v. 30, p. 229-240, 2017.

7. Federação Brasileira das associações de Síndrome de Down (FBASD). Síndrome de Down. Brasilia, 2018. Disponível em: < http://federacaodown.org.br/index.php/sindrome-de-down/ />. Acesso em: 18 dez. 2018.

8. GODZICKI B et al. Aquisição do sentar independente na Síndrome de Down utilizando o balanço. Fisioterapia em Movimento, v. 23, n. 1, 2017.

9. INSTITUTO BRASILEIRO DE GEOGRAFIA E ESTATÍSTICA (IBGE). Censo Demográfico: características da população e dos domicílios. Rio de Janeiro: Gráfica digital; 2010.

10. MENEGHETTI CHZ et al. Avaliação do equilíbrio estático de crianças e adolescentes com síndrome de Down. Brazilian Journal of Physical Therapy/Revista Brasileira de Fisioterapia, v. 13, n. 3, 2009. 
11. MOREIRA L et al. Down syndrome and its pathogenesis: considerations about genetic determinism. Revista Brasileira de Psiquiatria, v. 22, n. 2, p. 96-99, 2000.

12. MORAIS KDW et al. Profile of physiotherapy intervention for Down syndrome children. Fisioterapia em Movimento, v. 29, n. 4 , p. $693-701,2016$.

13. National Down Syndrome Society (NDSS). Down Syndrome. Washington, DC (EUA), 2018. Disponível em: < https://www.ndss.org/about-down-syndrome/down-syndrome/>. Acesso em: 18 dez. 2018.

14. PEREIRA WJG et al. Comunicação entre os Surdos e os Profissionais da Saúde, uma questão de saúde pública: Revisão Sistemática. III CONBRACIS (Congresso Brasileiro de Ciências da Saúde) Campina Grande -PB, v.1, 2018

15. PRIOSTI PA. et al. Força de preensão e destreza manual na criança com Síndrome de Down. 2009.

16. SANTOS JA et al.. Curvas de crescimento para crianças com Síndrome de Down. Rev Bras Nutr Clin, v. 21, n. 2, p. 144-8, 2006.

17. SCHWARTZMAN JS. et al. Síndrome de down. São Paulo: Mackenzie, v. 13, 1999

18. SHEPHERD R. Fisioterapia em Pediatria, 3를 edição, Santos Livraria Editora, 2002.

19. SILVA NLP e DESSEN MA. Síndrome de Down: etiologia, caracterização e impacto na família. Interação em psicologia, v. 6, n. 2, 2002.

20. TOBLE AM. et al. Hidrocinesioterapia no tratamento fisioterapêutico de um lactente com Síndrome de Down: estudo de caso. Fisioterapia em Movimento, v. 26, n. 1, 2017.

21. TORQUATO JA. et al. A aquisição da motricidade em crianças portadoras de Síndrome de Down que realizam fisioterapia ou praticam equoterapia. Fisioterapia em Movimento, v. 26, n. 3, 2017. 